\title{
Prevalence of Selected Cardiometabolic Risk Factors in The Global ART-Naïve HIV Infected Population: A Protocol for a Systematic Review and Meta-Analysis
}

Peter Vanes Ebasone ( $\sim$ petervanes2006@gmail.com )

University of Cape Town https://orcid.org/0000-0002-7342-5363

Nasheeta Peer

South African Medical Research Council

Anastase Dzudie

University of Yaounde I: Universite de Yaounde I

Andre Pascal Kengne

South African Medical Research Council

\section{Protocol}

Keywords: HIV, ART-naïve, cardiometabolic risk factors, prevalence

Posted Date: September 15th, 2021

DOI: https://doi.org/10.21203/rs.3.rs-868522/v1

License: (c) (1) This work is licensed under a Creative Commons Attribution 4.0 International License.

Read Full License 


\section{Abstract \\ Background}

People living with HIV/AIDS (PLHIV) are at increased risk of cardiometabolic diseases attributable to the effects of the virus, antiretroviral therapy (ART) and traditional risk factors found in the general population. Most studies have focused on assessing the effect of ART on cardiometabolic disease in PLHIV with fewer studies assessing the cardiometabolic risk profile prior to any exposure to ART. Therefore, this protocol is for a systematic review and meta-analysis to estimate the global prevalence of selected cardiometabolic risk factors in ART-naïve PLHIV and their association with HIV specific factors.

\section{Methods}

We shall conduct a systematic search of published literature for observational studies on the prevalence of obesity, hypertension, diabetes and dyslipidaemia (high Low-Density Lipoprotein Cholesterol, high Total Cholesterol, high Triglyceride, low High-Density Lipoprotein Cholesterol) in ART-naïve PLHIV and their association with HIV specific characteristics. We will search PubMed-MEDLINE, CINAHL, SCOPUS, Academic Search Premier, Africa-Wide Information and Africa Journals Online databases to identify relevant studies published before March 2021. Two authors will independently screen, select studies, extract data and conduct risk of bias assessments. Disagreements between the two authors will be resolved by consensus or consulting a third reviewer. Data consistently reported across studies will be pooled using random-effects meta-analysis. Heterogeneity will be evaluated using Cochrane's Q statistic and quantified using $\mathrm{I}^{2}$ statistics. The Preferred Reporting Items for Systematic reviews and MetaAnalysis protocols (PRISMA-P) 2015 guidelines are used for the reporting of this systematic review protocol.

\section{Discussion}

This review will help determine the burden of selected cardiometabolic diseases in ART-naïve HIV-infected populations and the contribution of HIV infection, independent of ART, to cardiometabolic diseases in PLHIV. It will provide new information that can help orientate future research and potentially guide healthcare policy making. This is part of a thesis that will be submitted to the Faculty of Health Sciences, University of Cape Town, for the award of a PhD in Medicine with protocol ethical clearance number (UCT HREC 350/2021).

\section{Registration}

PROSPERO: CRD42021226001 


\section{Background}

About 38 million people globally are infected with the Human Immunodeficiency Virus (HIV), most of whom reside in Sub-Saharan Africa (SSA) (1). The incidence of HIV remains high with 1.7 million new infections in 2018 alone (1). The introduction of potent antiretroviral therapy (ART), has witnessed a dramatic improvement in the quality of life with decreased morbidity, and longevity of people living with HIV/AIDS (PLHIV). Together with the high burden of HIV infection, worldwide, there is also a growing noncommunicable diseases (NCDs) epidemic including cardiometabolic diseases such as obesity, hypertension, type 2 diabetes mellitus (hereafter referred to as diabetes) and dyslipidaemia; responsible for increasing morbidity and mortality (2). With increased life expectancy following the introduction of ART, PLHIV are exposed to the same environmental and behavioural factors as general populations that drive cardiometabolic diseases risk. Therefore, the comprehensive management of PLHIV is shifting towards the co-management of NCDs including cardiometabolic diseases, other chronic diseases and cancers. The comorbidity of HIV and cardiometabolic diseases places a substantial strain on health care systems including increased healthcare costs and difficulties with optimal service delivery, particularly in resource constrained settings in low- and middle-income countries (LMICs) $(3,4)$.

HIV infection has been found to increase the risk for cardiometabolic diseases via the HIV virus itself and related opportunistic infections, as well as the effects of ART $(5,6)$. Several studies have shown that the risk of cardiometabolic disease is higher in PLHIV on ART than those who are ART-naïve and that the risk due to the virus itself is significantly higher than in the HIV negative population (7-9). As more PLHIV are living beyond 50 years of age $(10,11)$, they are more exposed to traditional risk factors such as ageing and a genetic predisposition together with lifestyle behaviours associated with unhealthy diets, physical inactivity, smoking and alcohol misuse, .

HIV accelerates and accentuates cardiometabolic changes by decreasing the body's regenerative ability, hence expediting the process of immunosenescence and systemic ageing $(3,4,12)$. Independent of ageing, HIV induces chronic inflammation, sustained immune activation and increases the risk of thrombosis $(13,14)$. Some studies have shown dyslipidaemia in the ART-naïve population, suggesting that the virus itself can cause lipid abnormalities including high triglycerides and low high-density lipoprotein cholesterol $(\mathrm{HDL}-\mathrm{C})(15,16)$. It has also been shown that through a similar pathway, HIV is a strong determinant of cardiovascular diseases such as myocardial infarction, stroke, and diabetes (1719).

As the uptake of ART becomes more widespread, there is an increased risk of cardiometabolic disease due to the direct effects of ART. Some ART regimens, especially protease inhibitors have been shown to be associated with increased levels of serum low-density lipoprotein cholesterol (LDL-C), triglycerides (TG), total cholesterol (TC) and insulin resistance $(5,19,20)$. Also, the new WHO recommended first line regimen Dolutegravir have been shown by early studies to be associated with significant increased body weight and an increased incidence of obesity $(21,22)$. 
Most studies have focused on the prevalence of cardiometabolic risk factors in PLHIV on ART. Fewer studies have explored cardiometabolic diseases in the ART-naïve HIV infected population. Particularly, the global prevalence of these cardiometabolic risk factors in the ART-naïve population is unknown. Assessing this prevalence will help inform and guide future research and public health interventions. Therefore, this study aims to assess the prevalence of obesity, hypertension, diabetes, and dyslipidaemia (high TC, high LDL-C, high TG, low HDL-C) in the global HIV infected ART-naïve population; and the association of HIV specific factors with these cardiometabolic risk factors.

\section{Review questions}

1. What is the prevalence of obesity, hypertension, diabetes, and dyslipidaemia (high TC, high LDL-C, high TG, low HDL-C) in the global ART-naïve PLHIV?

2. What is the association between HIV specific factors including time since diagnosis, severity of the disease, CD4 count and/or viral load and these selected cardiometabolic risk factors?

\section{Methods/design}

\section{Eligibility criteria}

\section{Inclusion criteria}

Studies involving human subjects and fulfilling the following criteria will be included:

1. Study designs: cross-sectional, case-control and cohort studies

2. Study participants: ART-naïve patients (aged 15 years or above), living with HIV/AIDS worldwide.

3. Clinical outcomes: the prevalence of any of the following: obesity, hypertension, diabetes or dyslipidaemia (high TC, high LDL-C, high TG, low HDL-C) or sufficient data for estimating the prevalence, with/without HIV specific factors (time since diagnosis, WHO stage, viral load and/or CD4 count).

4. Time-period: we will consider all published and unpublished data available up to the time of the review.

5. Study settings: health facilities or community-based settings.

6. Language: all studies reported in the English or French languages.

7. For studies reported more than once, the article with the largest number of participants will be included.

\section{Exclusion criteria}

Studies with the following characteristics will be excluded:

1. Studies lacking prevalence measurements or sufficient data to perform these estimates even after contacting the primary investigators. 
2. Case series, case reports, reviews, clinical trials, commentaries and editorials.

3. Studies not performed in human participants or published in languages other than English and French.

\section{Sources of Information}

The systematic review's methods are reported according to the Preferred Reporting Items for Systematic reviews and Meta-Analysis protocols (PRISMA-P) 2015 Guidelines, (see Additional file 1) (23).

\section{Search strategy for identifying studies}

\section{Electronic searches}

We will undertake a comprehensive electronic search across PubMed-MEDLINE, CINAHL, SCOPUS, Academic Search Premier, Africa-Wide Information and Africa Journals Online databases to identify relevant studies. The search shall be conducted using a predefined comprehensive and sensitive search strategy that will comprise combinations of MESH terms, CINAHL headings, and free words relating to cardiometabolic risk factors, ART-naïve and HIV/AIDS (Table 1). We will further use controlled vocabularies synonyms to identify related terms. 
Table 1

PubMed-MEDLINE Search Strategy

\section{Search Search Terms}

1 Cardiometabolic diseases[tw] OR Cardiometabolic disease[tw] OR Cardiometabolic syndrome[tw] OR Cardiometabolic traits[tw] OR Cardiometabolic risk factors[tw] OR Cardiometabolic factors[tw] OR cardiovascular[tw] OR Metabolic Syndrome[tw] OR Metabolic Syndrome X[MeSH Terms] OR Reaven Syndrome[tw] OR syndrome X[tw]

2 Obesity[MeSH Terms] OR obesity[tw] OR obese[tw] OR overweight[tw] OR adiposity[tw] OR body mass index[tw] OR BMI[tw] OR Waist circumference[tw] OR waist [tw] OR hip circumference[tw] OR waist-to-hip ratio[tw] OR waist-to-height ratio[tw]

Hypertension[MeSH Terms] OR High blood pressure [tw] OR raised blood pressure[tw] OR Blood pressure[tw] OR systolic blood pressure[tw] OR diastolic blood pressure[tw] OR SBP[tw] OR DBP [tw] OR elevated blood pressure [tw]

4

Diabetes mellitus[MeSH Terms] OR diabetes[tw] OR diabetes mellitus[tw] OR type 2 diabetes mellitus[tw] OR type 2 diabetes[tw] OR glucose[tw] OR hyperglycemia[tw] OR hyperglycaemia[tw] OR Glycated haemoglobin[tw] OR HbA1c[tw] OR Impaired fasting glucose[tw] OR Fasting glucose[tw] OR dysglycemia[tw] OR dysglycaemia[tw] OR Glycated hemoglobin[tw]

Dyslipidemia[MeSH Terms] OR dyslipidaemia[tw] OR dyslipidemia[tw] OR lipids[tw] OR lipid[tw] OR cholesterol[tw] OR hyperlipidemia[tw] OR hyperlipidaemia[tw] OR hypercholesterolemia[tw] OR hypertriglyceridemia[tw] OR triglyceride[tw] OR triglycerides[tw] OR total cholesterol[tw] OR HDL[tw] OR LDL[tw] OR TG[tw] OR TCtw] OR VLDL[tw] OR hyperlipoproteinemia[tw] OR hyperlipidaemia[tw] OR Lipid disorder[tw] OR HDL cholesterol[tw] OR high-density lipoprotein cholesterol[tw] OR LDL cholesterol [tw] OR low-density lipoprotein cholesterol[tw] OR HDL-C[tw] OR LDLC[tw] OR hyperlipidaemia[tw]

ART-naive[tw] OR ART naive[tw] OR untreated[tw] OR Antiretroviral therapy naive[tw] OR Non-ART[tw] OR Non-ART users[tw] OR ART-unexposed[tw] OR HAART-naive[tw] OR HAART naive[tw] OR HAART-untreated[tw] OR HAART unexposed[tw] OR ARVunexposed[tw]

7

HIV[MeSH Terms] OR HIV[tw] OR HIV/AIDS[tw] OR human immunodeficiency virus[tw] OR acquired immunodeficiency syndrome[tw] OR AIDS[tw] OR acquired immunodeficiency syndrome[MeSH Terms]

\section{Grey literature}

We will search reference lists of relevant citations via Web of Science and scan the reference lists of review papers and conference proceedings. We will also examine publications on the websites of key organisations such as UNAIDS, WHO, and the International AIDS Society. Key experts in the field will be contacted for any unpublished study. 


\section{Study Records}

\section{Data management}

Search results shall be collected into EPPI-Reviewer software for de-duplication. Well-structured and standardized questions developed in accordance to the inclusion criteria shall be used for screening studies in EPPI-Reviewer.

\section{Screening}

Two investigators will independently screen titles and abstracts using the aforementioned inclusion criteria. Full texts will then be obtained and screened using a standardised and pre-tested form to include eligible studies. Disagreements will be resolved by consensus or by consulting a third investigator. We will contact corresponding authors of potentially eligible studies for relevant data that were not reported. Reasons for exclusion of non-eligible studies will be documented. The whole selection process will be summarised in a PRISMA flow chart (Additional File 2).

\section{Data extraction}

The data for this review will be extracted using a purposeful design and piloted extraction form designed on LimeSurvey. Two investigators will independently extract data from included studies and any inconsistencies or disagreement shall be resolved by consensus or consultation with the third investigator.

\section{Data Items}

The data to be extracted will include 1) Author and paper details [first author name, year of publication, language of publication]; 2) Study characteristics [country, study design, coverage (national or sub national), study setting (urban vs rural or both), study period, sampling method, participants age limit, sample size, response rate]; 3) Participants' characteristics [mean or median age and age range, gender (proportion of males), employment status, level of education, lifestyle habits (smoking, alcohol misuse), 4) HIV-related factors (time since diagnosis, severity of the disease, CD4 count and/or viral load, mean/median CD4, proportion with CD4 count $<200$, median duration of HIV or on ART, ART regimens); and 5) Prevalence measures [number of participants with CMRFs of interest and definition of each CMRF]. 6) Association of HIV specific factors and CMRFs (measure of association, effect size estimate, standard error of effect size and adjustment for confounders)

\section{Assessment of methodological quality and risk of bias}

Two reviewers will independently score the quality of the included studies. For each study, the risk of bias and evaluation of methodological quality will be checked using the risk of bias tool for prevalent studies checklist adopted from Hoy et all (24) (Table 2). The overall risk of bias will be scored and summarised as low, intermediate or high risk. Assessment of the risk of selection and attrition bias will use the 
Cochrane guidelines available in Review Manager V.5.4.1 (http://tech.cochrane.org/revman). Any discrepancies will be resolved by consensus or by consulting the third investigator. Inter-rater agreement on screening, data extraction and methodological quality will be assessed using Cohen's $\mathrm{K}$ coefficient (25). The risk of bias and quality scores will be presented in a table. 
Table 2

Quality assessment checklist for prevalence studies

\section{Name of author(s):}

Year of publication:

Study title:

Risk of bias item

External validity

1 Was the study target population a close representation of the national population in relation to relevant variables?

2

Was the sampling frame a true or close representation of the target population?

3

Was some form of random selection used to select the sample, OR, was a census undertaken?

4

Was the likelihood of non-participation bias minimal?

Internal validity

5

Were data collected directly from the participants (as opposed to medical records)?

6

Were acceptable case definitions used in the study?

7

Were reliable and accepted diagnostic methods for diagnosing cardiometabolic risk factors of interest utilised?

8

Was the same mode of data collection used for all participants?

9

Were the numerator(s) and denominator(s) for the calculation of the cardiometabolic risk factors of interest appropriate?

Summary of the overall risk of bias
Low risk $0-$ 3

Intermediate risk $4-$ 6

High risk

Data synthesis, analysis and assessment of heterogeneity 
A summary of prevalence data will be presented by country and continent. Where population data is reported at both continental and country levels, we shall pick the most comprehensive and updated national estimates. Other materials will then be treated as duplicates and consequently excluded. Metaanalysis and meta-regression analysis will be conducted for identical variables across studies. For studies with sufficient data, a meta-analysis for the prevalence and associations will be conducted across all eligible studies using random effects models. The study-specific estimates will be pooled through a random-effects meta-analysis model, to obtain the overall summary estimate of the prevalence and associations across studies, after stabilising the variance of individual studies with the use of FreemanTukey double arc-sine transformation (26). This transformation will help reduce the effect of extremely high or extremely low measurement values on the pooled estimate. Heterogeneity will be evaluated by the Cochrane's Q statistic and $\mathrm{I}^{2}$. $\mathrm{I}^{2}$ values of $25 \%, 50 \%$ and $75 \%$ will be deemed to represent low, medium and high heterogeneity, respectively. Funnel plots together with the Egger test of bias will be used to investigate the publication bias (27).

We will also conduct subgroup analyses to compare the estimates across major predictive characteristics and assess the consistency of the effects across those subgroups. Major grouping characteristics will include gender (gender-specific analysis where possible; and below vs. at or above the median proportion of men across study); age group (below vs. at or above the median), geographic region, time the study was conducted/published (below vs. at or above the median); sample size (below vs. at or above median sample size across included studies); severity of disease (WHO stage at diagnosis); study design, etc. We will report the total number of relevant factors investigated across all studies, and for each factor, the number of times it was reported to be associated with the outcome. We will further report on the measure of association used for each factor across studies, with indication of whether those measures were adjusted for confounders or not.

Data analyses will use the 'meta' package of the latest version of R statistical software (R Foundation for statistical computing, Vienna, Austria).

\section{Sensitivity analysis}

A sensitivity analysis will help evaluate the extent to which the meta-analytical results and conclusions are altered by changes in analysis approach. We will use the leave-one-out jackknife sensitivity analysis in which one primary study is excluded at a time. This will help us assess if specific studies are influencing the overall estimates. We will then compare the new pooled prevalence or effect size estimate with that of the original one. If the new pooled prevalence or effect size measurement lies outside of the $95 \%$ Confidence Interval $(\mathrm{Cl})$ of the original pooled prevalence, we will conclude that the excluded study has a significant effect and should be excluded from the final analysis

The Duval and Tweedie trim-and-fill will be used to adjust estimates for the effects of potential publication bias.

\section{Reporting of this review}


The proposed systematic review will be reported following the PRISMA guidelines (28). We intend to publish a PRISMA checklist alongside the final report.

\section{Potential amendments}

We do not intend to make any amendments to the protocol, to avoid the possibility of outcome reporting bias. However, any amendments that do prove necessary will be documented and reported transparently.

\section{Discussion}

Since cardiometabolic diseases in PLHIV are driven by HIV infection per se and ARTs, together with traditional risk factors, it is important to understand the differential contribution and distribution of cardiometabolic risk factors among the HIV-infected who are not yet exposed to ARTs. This review will help determine the burden of selected cardiometabolic diseases in ART-naïve HIV-infected populations and the contribution of HIV infection, independent of ART, to cardiometabolic diseases in PLHIV. Since most published studies have largely focused on measuring the prevalence of cardiometabolic diseases and the associated impact of ARTs in PLHIV, it is important to estimate this prevalence in the ART-naïve PLHIV worldwide. This study therefore aims to provides new information that can help orientate future research and potentially guide healthcare policy making. If a high prevalence of cardiometabolic disease among ART-naïve PLHIV is demonstrated, this may indicate an increased need to strengthen efforts for earlier screening, diagnosis and treatment of cardiometabolic diseases in PLHIV.

The selection of studies published in the English or French languages only may prevent relevant data from studies published in other languages from being included. This review will, however, identify gaps in the current literature on this topic and provide direction for future research in ART-naïve PLHIV.

\section{Abbreviations}

ART

Antiretroviral therapy

Cl

Confidence Interval

HDL-C

High-Density Lipoprotein Cholesterol

HIV

Human Immunodeficiency Virus

LDL-C

Low-Density Lipoprotein Cholesterol

LMICs

Low- and Middle-Income Countries

NCDs

Page $11 / 15$ 
Non-Communicable Diseases

PLHIV

People living with HIV

PRISMA-P

Preferred Reporting Items for Systematic reviews and Meta-Analysis protocols

SSA

Sub-Saharan Africa

STROBE

Report of methodology will be assessed using the Strengthening the Reporting of Observational Studies in Epidemiology

TC

Total Cholesterol

TG

Triglycerides

UNAIDS

The Joint United Nations Programme on HIV/AIDS

WHO

World Health Organisation

\section{Declarations}

\section{Ethics approval and consent to participate}

Consent to participate will not be needed for this study as data used will be extracted from already published studies. This review is part of a thesis that will be submitted to the Faculty of Health Sciences, University of Cape Town, for the award of a PhD in Medicine. It was approved by the University of Cape Town Human Research Ethics Committee- IRB0001938 (UCT HREC REF: 350/2021).

\section{Consent for publication}

Not applicable

\section{Availability of data and materials}

The datasets used and/or analysed during the current study will be available from the corresponding author on reasonable request.

\section{Competing interests}

The authors declare that they have no competing interests.

\section{Funding}


This project is part of the EDCTP2 programme supported by the European Union (grant number TMA2017GSF-1962 - CaDERAL) through the South African Medical Research Council. APK and NP are employed by the South African Medical Research Council. The Sponsors have no role in designing and conducting of this study.

\section{Author's contributions}

PVE, APK and NP conceived and designed the review. APK, NP, AD provided general guidance for the drafting of the protocol. PVE drafted the manuscript and is the guarantor this review. APK, NP and AD reviewed and revised the manuscripts. All authors have read and approved the final version of the manuscript.

\section{Acknowledgements}

Not applicable

\section{References}

1. UNAIDS D, Update AE. Geneva. Joint United Nations Programme on HIV. AIDS. 2019.

2. Kassa M, Grace J. The Global Burden and Perspectives on Non-Communicable Diseases (NCDs) and the Prevention, Data Availability and Systems Approach of NCDs in Low-resource Countries. Public Health Dev Ctries - Chall Oppor [Internet]. 2019 Nov 8 [cited 2020 Oct 23]; Available from: https://www.intechopen.com/books/public-health-in-developing-countries-challenges-andopportunities/the-global-burden-and-perspectives-on-non-communicable-diseases-ncds-and-theprevention-data-availab.

3. Althoff KN, Smit M, Reiss P, Justice AC. HIV and Ageing: Improving Quantity and Quality of Life. Curr Opin HIV AIDS. 2016 Sep;11(5):527-36.

4. Appay V, Kelleher AD. Immune activation and immune aging in HIV infection. Curr Opin HIV AIDS. 2016 Mar;11(2):242-9.

5. Kiage JN, Heimburger DC, Nyirenda CK, Wellons MF, Bagchi S, Chi BH, et al. Cardiometabolic risk factors among HIV patients on antiretroviral therapy. Lipids Health Dis. 2013;12:50.

6. Nguyen KA, Peer N, Mills EJ, Kengne AP. A Meta-Analysis of the Metabolic Syndrome Prevalence in the Global HIV-Infected Population. PLoS ONE [Internet]. 2016 [cited 2020 Oct 23];11(3). Available from: https://www.ncbi.nlm.nih.gov/pmc/articles/PMC4805252/.

7. Obel N, Thomsen HF, Kronborg G, Larsen CS, Hildebrandt PR, Sørensen HT, et al. Ischemic Heart Disease in HIV-Infected and HIV-Uninfected Individuals: A Population-Based Cohort Study. Clin Infect Dis. 2007 Jun 15;44(12):1625-31.

8. Islam FM, Wu J, Jansson J, Wilson DP. Relative risk of cardiovascular disease among people living with HIV: a systematic review and meta-analysis. HIV Med. 2012;13(8):453-68. 
9. Botha S, Fourie CMT, Rooyen JM van, Kruger A, Schutte AE. Cardiometabolic Changes in Treated Versus Never Treated HIV-Infected Black South Africans: The PURE Study. Heart Lung Circ. 2014 Feb 1;23(2):119-26.

10. Nyirenda M, Newell M-L, Mugisha J, Mutevedzi PC, Seeley J, Scholten F, et al. Health, wellbeing, and disability among older people infected or affected by HIV in Uganda and South Africa. Glob Health Action. 2013 Dec;1(1):19201. 6(.

11. Negin J, Martiniuk A, Cumming RG, Naidoo N, Phaswana-Mafuya N, Madurai L, et al. Prevalence of HIV and chronic comorbidities among older adults. AIDS Lond Engl. 2012 Jul 31;26(0 1):S55-63.

12. Appay V, Sauce D. Immune activation and inflammation in HIV-1 infection: causes and consequences. J Pathol. 2008 Jan;214(2):231-41.

13. Cerrato E, Calcagno A, D’Ascenzo F, Biondi-Zoccai G, Mancone M, Marra WG, et al. Cardiovascular disease in HIV patients: from bench to bedside and backwards. Open Heart. 2015 Mar 1;2(1):e000174.

14. Mayne ES, George JA. Mortal allies: human immunodeficiency virus and noncommunicable diseases. Curr Opin HIV AIDS. 2017 Mar;12(2):148-56.

15. Souza SJ, Luzia LA, Santos SS, Rondó PHC. Lipid profile of HIV-infected patients in relation to antiretroviral therapy: a review. Rev Assoc Medica Bras 1992. 2013 Apr;59(2):186-98.

16. Grunfeld C, Pang M, Doerrler W, Shigenaga JK, Jensen P, Feingold KR. Lipids, lipoproteins, triglyceride clearance, and cytokines in human immunodeficiency virus infection and the acquired immunodeficiency syndrome. J Clin Endocrinol Metab. 1992 May;74(5):1045-52.

17. Syed FF, Sani MU. Recent advances in HIV-associated cardiovascular diseases in Africa. Heart. 2013 Aug 15;99(16):1146-53.

18. Brown TT, Cole SR, Li X, Kingsley LA, Palella FJ, Riddler SA, et al. Antiretroviral therapy and the prevalence and incidence of diabetes mellitus in the multicenter AIDS cohort study. Arch Intern Med. 2005 May 23;165(10):1179-84.

19. Tsiodras S, Mantzoros C, Hammer S, Samore M. Effects of protease inhibitors on hyperglycemia, hyperlipidemia, and lipodystrophy: a 5-year cohort study. Arch Intern Med. 2000 Jul;10(13):2050-6. 160 .

20. Maggi P, Di Biagio A, Rusconi S, Cicalini S, D’Abbraccio M, d'Ettorre G, et al. Cardiovascular risk and dyslipidemia among persons living with HIV: a review. BMC Infect Dis [Internet]. 2017 Aug 9 [cited 2020 Oct 23];17. Available from: https://www.ncbi.nlm.nih.gov/pmc/articles/PMC5550957/.

21. Dolutegravir-Based or. Low-Dose Efavirenz-Based Regimen for the Treatment of HIV-1. N Engl J Med. 2019 Aug;29(9):816-26. 381(.

22. Venter WDF, Moorhouse M, Sokhela S, Fairlie L, Mashabane N, Masenya M, et al. Dolutegravir plus Two Different Prodrugs of Tenofovir to Treat HIV. N Engl J Med. 2019 Aug;29(9):803-15. 381(.

23. Moher D, Shamseer L, Clarke M, Ghersi D, Liberati A, Petticrew M, et al. Preferred reporting items for systematic review and meta-analysis protocols (PRISMA-P) 2015 statement. Syst Rev [Internet]. 2015 [cited 2020 Oct 18];4(1). Available from: https://www.ncbi.nlm.nih.gov/pmc/articles/PMC4320440/. 
24. Hoy D, Brooks P, Woolf A, Blyth F, March L, Bain C, et al. Assessing risk of bias in prevalence studies: modification of an existing tool and evidence of interrater agreement. J Clin Epidemiol. 2012 Sep 1;65(9):934-9.

25. McHugh ML. Interrater reliability: the kappa statistic. Biochem Medica. 2012 Oct;15(3):276-82. 22(.

26. Barendregt JJ, Doi SA, Lee YY, Norman RE, Vos T. Meta-analysis of prevalence. J Epidemiol Community Health. 2013 Nov;1(11):974-8. 67(.

27. Egger M, Smith GD, Schneider M, Minder C. Bias in meta-analysis detected by a simple, graphical test. BMJ. 1997 Sep;13(7109):629-34. 315(.

28. Moher D, Liberati A, Tetzlaff J, Altman DG. Preferred Reporting Items for Systematic Reviews and Meta-Analyses: The PRISMA Statement. Ann Intern Med. 2009 Aug;18(4):264-9. 151 (.

\section{Supplementary Files}

This is a list of supplementary files associated with this preprint. Click to download.

- Additionalfile1.pdf

- Additionalfile2.pdf 